\title{
Health-related quality of life in Croatian general population and multiple myeloma patients assessed by the EORTC QLQ-C30 and EORTC QLQ-MY20 questionnaires
}

\author{
Sanja Ledinski Ficko1,2, Vlatko Pejsa ${ }^{3}$, Vesna Zadnik ${ }^{4}$ \\ ${ }^{1}$ University of Applied Health Sciences, Department of Nursing, Zagreb, Croatia \\ ${ }^{2}$ University of Ljubljana, Interdisciplinary Doctoral Study of Biomedicine, Ljubljana, Slovenia \\ ${ }^{3}$ Clinical Hospital Dubrava, Institut of Hematology, Zagreb, Croatia \\ ${ }^{4}$ Institute of Oncology Ljubljana, Epidemiology and Cancer Registry, Ljubljana, Slovenia
}

Radiol Oncol 2019; 53(3): 337-347

Received 23 July 2019

Accepted 9 August 2019

Correspondence to: Assoc. Prof. Vesna Zadnik, M.D., Ph.D., Institute of Oncology Ljubljana, Epidemiology and Cancer Registry, Zaloška cesta 2, SI-1000 Ljubljana, Slovenia. Phone: + 38615879 451; E-mail: vzadnik@onko-i.si

Disclosure: No potential conflicts of interest were disclosed.

Background. The impact of disease and treatment on the patient's overall well-being and functioning is a topic of growing interest in clinical research and practice. The aim of this study is to obtain reference data on quality of life of Croatian general population. Further, we aim to assess the impact of the disease and its primary systemic treatment on their health related quality of life (HrQoL) in multiple myeloma (MM) patients.

Patients and methods. Participants for the first part of the study were randomly selected from adult Croatian population. In the clinical part of the study MM patients were included as prospectively diagnosed within two years in two major Croatian haematological centres. The EORTC QLQ-C30 in both trials and QLQ-MY20 in MM patients only were applied for HrQoL assessment.

Results. Gender, age and place of residence have great impact on quality of life scores in Croatian population. The MM patients at the time of diagnosis have lower QLQ-C30 scores for global quality of life, functional and symptom scale scores, as well as single items. The type of disease followed by the choice of therapy options are important HrQoL determinants.

Conclusions. The norm values available now for Croatian population will help to interpret HrQoL for clinicians and aid in planning cancer care interventions. This study identified treatment effect consistent with those from other observational studies and provided new data on HrQoL across two different treatment choices for MM patients.

Key words: health-related quality of life; multiple myeloma; EORTC QLQ-C30; EORTC MY-20; reference data; Croatian population

\section{Introduction}

Cancer incidence is increasing both in developed and developing countries. According to the Croatian Institute for Public Health, in Croatia cancer incidence has been steadily rising from 1990 on. In 2016, there were 23,650 newly diagnosed cancer cases, less than 1 percent are younger than 20 years at diagnoses. Among adult patients 3 to 4 percent are diagnosed before the age of $40 .{ }^{1}$ Statistic data of the International Agency for Research on Cancer show that the incidence of multiple myeloma (MM) in Croatia for men is 4.0 with mortality of 2.1 in 100,000 citizens, and for women the incidence is 3.4 with mortality of 2.1 in 100,000 citizens. This means that every year we have 225-260 new MM cases in Croatia. MM is more frequent in males; the incidence increases with age. ${ }^{1}$ In 2016, there were 
no case of MM before the age of 40 in Croatia, and most patients are aged 70 years or more at the time of diagnosis. ${ }^{1}$ Given the number of patients, there is a great need for assessing the health-related quality of life (HrQoL) to improve the care of oncology patients. Therefore, quality of life assessment is becoming more common in oncology. ${ }^{2}$

Health is one of the most important variables affecting well-being. ${ }^{3}$ World Health Organisation defines quality of life as the individuals' perception of their position in life in the context of the culture and value system in which they live and in relation to their goals, expectations, standards and concerns. ${ }^{4}$ The impact of disease and treatment on the patient's overall well-being and functioning is a topic of growing interest in clinical research and practice. ${ }^{5}$ Indicators of quality of life can provide evidence that is based on comparable and standardized measures and that can be used to improve the health care system. Conducting studies using various health-related quality of life measures is a basis for improvement of health care delivery. ${ }^{6}$ In the last decades, the measurement of healthrelated quality of life has gained acceptance as a primary or secondary endpoint in cancer research worldwide. ${ }^{7}$

Evaluation of quality of life is conducted by using standardized questionnaires. ${ }^{8}$ European Organization for Research and Treatment of Cancer (EORTC) has purposed a development of an integrated, modular approach for evaluating the quality of life of cancer patient. They have launched a core EORTC quality of life questionnaire for cancer patients that consists of 30 questions (EORTC QLQ-C30). The EORTC QLQ-C30 is one of the most widely used instrument for assessing healthrelated quality of life in cancer patients ${ }^{9}$ and has been used so far in more than 3000 studies worldwide. The core questionnaire is supplemented by disease-specific modules. ${ }^{10}$ So far disease specific modules were developed for 13 different malignant conditions. The EORTC QLQ-MY20 is specific questionnaire adjusted for patients with multiple myeloma. The EORTC QLQ questionnaires have been translated and validated into 81 languages. The EORTC QLQ-C30 and EORTC QLQ-MY20 exist also in Croatian language. ${ }^{11,12}$

The normative (reference) values of QLQ-C30 questionnaire for general healthy population are already available for some countries, for example Germany $^{5}$, the Netherlands ${ }^{13}$, Denmark ${ }^{7}$, Sweden ${ }^{14}$ and Slovenia. ${ }^{15}$ Typically, they demonstrate the decrease of HrQoL on all levels with age in both sexes. On the contrary, some symptoms such as pain and fatigue increase with age. The population norms are applied in clinical practice as an aid to the clinical assessment of an individual patient and in research to assist in the overall interpretation of results from clinical studies of HrQoL.

The knowledge on HrQoL in MM patients is scarce. The findings of the recent European cohort study provide a better understanding on how to improve the treatment of patients with MM in order to improve their QoL. The data indicated that there is a need for better management of the treatment of fatigue and bone related symptoms since those are the strongest HrQoL predictors. The study also suggested that specific QoL aspects can be notably improved by treatment which targets specific symptoms. ${ }^{16}$

The aim of this study is to obtain reference data on quality of life in a representative sample of Croatian general population older than 40 years, and obtaining data on quality of life in MM patients with the aid of EORTC QLQ-C30 and QLQ-MY20 questionnaires. The norm values will help to interpret health-related quality of life data for clinicians, and aid in planning interventions for symptoms in the early stages of the disease. In MM patients, we aim to assess the impact of the disease and its primary systemic treatment on their HrQoL.

\section{Patients and methods}

\section{Population sample}

The study was conducted in the second half of 2016 and during 2017. For the initial part of our study, eligible respondents were adults, aged over 40 at the entry, residents of Croatia with no history of a malignant disease. A random sample of adult inhabitants in all six regions of Croatia: Dalmatia, Slavonia, Istria, Podravina, Zagreb region and Medimurje has been obtained. The EORTC QLQ-C30 questionnaire supplemented by a demographic inquiry was personally delivered to randomly selected individuals. The data collection was carried out by specially trained medical nurses in health centres, homes for the elderly, in the street and in other highly frequented places. For any randomly chosen adult we firstly determined their age and health status, and, in the case of not having a malignant disease and being older than 40 , we continued with collecting the quality of life data. From the 362 initially contacted, 310 respondents were included in our population sample. Of 51 not included respondents, 35 had cancer, while 16 of them chose not to participate for different 
personal reasons. The Survey Monkey Sample Size Calculator ${ }^{17}$ was used to estimate that such a sample size is sufficient for our study to reach adequate power.

\section{Multiple myeloma patient sample}

In the second part of the study MM patients older than 40 were included. A prospective study was conducted in the second half of 2016, 2017 and the first half of 2018. EORTC QLQ-C30 and EORTC QLQ-MY20 questionnaires were filled in by respondents before and three months after treatment with chemotherapy and stem cell transplantation in two major haematological centres in Croatia: Clinical Hospital Dubrava and Clinical Hospital Merkur. Questionnaires were distributed to the respondents during their stay in hospital, at the moment when transplantation or chemotherapy is determined as a therapeutic procedure. The second evaluation was conducted three months after therapeutic procedures. The sample consisted of 25 respondents before and after stem cell transplantation and 26 respondents before and after chemotherapy, which corresponds to a similar Czech research which evaluated the quality of life among malignant lymphoma and MM patients undergoing autologous stem cell transplantation. ${ }^{16}$ Additional five people were invited to the study but they chose not to participate.

\section{Questionnaires}

In both samples we used the EORTC QLQ-C30 questionnaire which contains 30 questions. The official Croatian translation of the questionnaire was used in assessing individual HrQoL during previous week. Respondents were offered four response alternatives: 1 "not at all", 2 "a little", 3 "quite a bit" and 4 "very much". In the last two questions the range was 1 to 7 in which 1 is "very poor" and 7 is "excellent". The EORTC QLQ-C30 questionnaire consists of a general health/quality of life scale, and five functional scales: physical functioning, role functioning, cognitive functioning, emotional functioning and social functioning, and 13 symptom items. Functional scale includes cognitive, emotional, physical, role and social functioning dimension. Symptom scale includes fatigue, nausea or vomiting and pain, and single items include loss of appetite, constipation, diarrhoea, dyspnoea, financial impact and sleep disturbance. For general quality of life and functional scale, higher scores mean better quality of life while higher scores for symptom scale and single items mean lower quality of life. ${ }^{18}$ In addition to EORTC QLQ-C30 all the participants also responded to a questionnaire on their demographic data including age, gender and place of residence.

The EORTC QLQ-MY20 is specific questionnaire adjusted for patients with multiple myeloma. The official Croatian translation of the questionnaire was used in assessing individual HrQoL during the previous week. It consists of functional scales which include future perspective and body image, and symptom scale which includes disease symptoms and side effects of treatment. Respondents were offered four response alternatives: 1 "not at all", 2 "a little", 3 "quite a bit" and 4 "very much". The higher the number of an item means poorer functioning. ${ }^{18}$

\section{Internal consistency}

Some dimensions are composed of more than one answer, and the others include only one categorical answer. Internal consistency of multiple answers within single dimension was proved by using the Cronbach alpha coefficient of reliability. The high value of the Cronbach alpha coefficients suggests a very high reliability. The coefficients were as follows: general health status/quality of life $(0,87)$, physical functioning $(0,84)$, role functioning $(0,86)$, emotional functioning $(0,87)$, cognitive functioning $(0,70)$, social functioning $(0,88)$, fatigue $(0,83)$, nausea/vomiting $(0,69)$ and pain $(0,81)$. In case of a lack of response to a question, the average of the other answers was used, but only if at least half of the questions were answered. ${ }^{19-21}$

To investigate if there is a correlation between EORTC QLQ-C30 and QLQ-MY20 scores, Pearson correlation coefficient calculations were performed.

\section{Statistical analysis}

Demographic characteristics were analysed as categorical variables and are represented in numbers and relative frequencies. Four age categories (40-49, 50-59, 60-69 and 70+) and six geographical regions were applied. The chi-square test was applied for determining the statistical significance of difference in demographical characteristics among groups of individuals in the sample. The answers, which were recorded in the EORTC QLQ-C30 and EORTC QLQ-MY20 were converted into dimensions, which evaluate the quality of life associated with health. Dimensions are ranged from 0-100 according to the EORTC scoring instructions. ${ }^{18}$ 


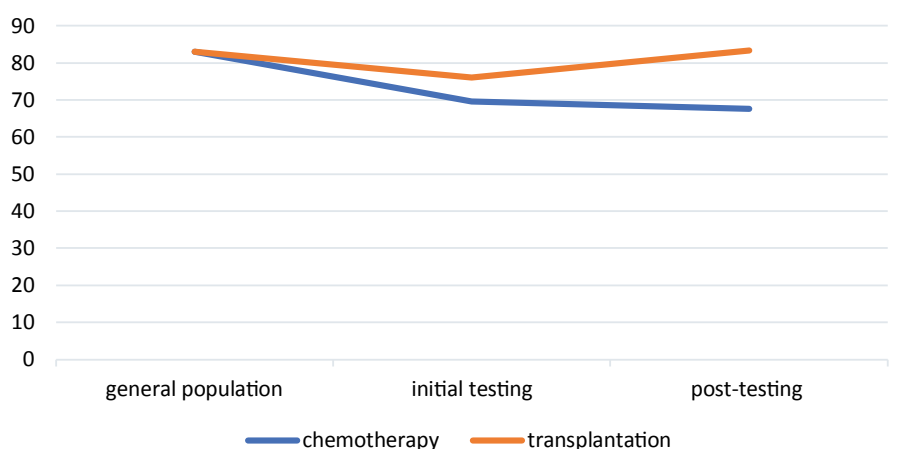

FIGURE 1. Summary score for EORTC QLQ-C30 in general population, initial testing and post-testing.

Dimensions are described by the arithmetic mean and standard deviation. As suggested by Nielsen et al. ${ }^{22}$ Mann-Whitney U test was used to compare the dimensions within two groups (gender, treatment). Where there were more than two groups for comparison (age, region) we used the KruskalWallis test. For testing the statistical significance of the change in dimensions before and after treatment Wilcoxon signed rank test was used.

Furthermore, for the first time to the best of our knowledge, the QLQ-C30 summary score of the EORTC QLQ-C30 was calculated for the popula- tion reference scores. QLQ-C30 summary score is a single higher-order model based on 27 of the 30 items of the QLQ-C30, excluding global quality of life and financial difficulties proposed by Giesinger et al..$^{23}$ In our study we investigated the ability of the QLQ-C30 summary score to distinguish between groups formed according to treatment choice (chemotherapy, transplantation) and general population.

The values of $p<0.05$ were evaluated as statistically significant. Statistical analysis was performed using the 24.0 SPSS Inc., Chicago, IL, SAD software.

\section{Compliance with ethical standards}

The data collection, preparation, implementation and presentation were in accordance with legal requirements for protecting the confidentiality of personal information: in Croatia non-drug trials are reviewed by ethics committees at an institutional level. Approval for the study was obtained from the Medical Ethics Committee of the Clinical Hospital Dubrava and Clinical Hospital Merkur (03/1-6234). All participants were fully informed about the aims of the study and provided written informed consent for participation in the study.

TABLE 1. Mean score (MS) and standard deviations (SD) for all EORTC QLQ-C30 dimensions and scales by age for general population

\begin{tabular}{|c|c|c|c|c|c|c|c|c|c|}
\hline & \multicolumn{2}{|c|}{$40-49$} & \multicolumn{2}{|c|}{$50-59$} & \multicolumn{2}{|c|}{$60-69$} & \multicolumn{2}{|c|}{70 and older } & \multirow{2}{*}{$\mathrm{p}^{*}$} \\
\hline & MS & SD & MS & SD & MS & SD & MS & SD & \\
\hline Global health status/quality of life & 69.1 & 18.6 & 71.1 & 20.7 & 67.4 & 19.9 & 56.9 & 23.9 & .035 \\
\hline Role functioning & 86.1 & 20.6 & 85.9 & 20.2 & 80.8 & 28.6 & 69.1 & 37.2 & .128 \\
\hline Emotional functioning & 71.9 & 22.4 & 78.2 & 19.7 & 78.6 & 19.7 & 66.7 & 26.4 & .034 \\
\hline Social functioning & 86.8 & 20.1 & 90.6 & 15.5 & 88.5 & 20.8 & 76.5 & 31.0 & .049 \\
\hline Fatigue & 33.7 & 20.8 & 28.0 & 22.7 & 29.5 & 22.8 & 43.8 & 29.2 & .014 \\
\hline Nausea/vomiting & 6.9 & 14.2 & 4.9 & 11.3 & 4.6 & 10.4 & 10.3 & 20.1 & .276 \\
\hline Pain & 25.8 & 24.3 & 23.3 & 24.5 & 24.1 & 27.8 & 40.2 & 32.3 & .028 \\
\hline Dyspnoea & 18.8 & 24.6 & 17.3 & 23.6 & 22.1 & 25.9 & 26.5 & 30.6 & .362 \\
\hline Diarrhoea & 7.9 & 15.8 & 6.5 & 13.9 & 6.2 & 13.0 & 13.7 & 26.1 & .595 \\
\hline Financial difficulties & 7.6 & 17.7 & 7.4 & 17.7 & 14.4 & 26.9 & 22.5 & 33.6 & .009 \\
\hline Summary score & 83.1 & 12.5 & 85.8 & 12.2 & 82.9 & 15.0 & 72.6 & 23.7 & .030 \\
\hline
\end{tabular}

$*^{p}=$ Kruskal-Wallis test 


\section{Results}

From the general population there were 310 questionnaires eligible for analysis; $68.4 \%$ women (212 persons) and $31.6 \%$ men (98 persons). The largest number of the respondents were in the age range of $50-59$ years, at $36.8 \%$ (114 persons). Most of the respondents were from the Zagreb region, at 33.9\% (105 persons). Furthermore, $51 \mathrm{MM}$ patients were included in the second part of the research. The largest number of the MM respondents were in the age range of 60-69 years, 20 patients. 25 of the respondents were treated with chemotherapy while 26 of the respondents underwent transplantation. Supplement table 1 shows how the groups differ in their demographic characteristics.

\section{Scale and item scores}

Results illustrate the transformed values of general quality of life, functional and symptom scales in the range 1-100. Table 1 shows the results for the EORTC QLQ-C30 for all scales, single items, as well as the summary score in general population according to age. In many dimensions there is a statistically significant difference in age in our sample. The scores for general health status are highest for the respondents aged 50-59 and lowest for the respondents who are 70 and older. Furthermore, there is also a statistically significant difference according to age for physical functioning, emotional functioning and social functioning. The scores are significantly lower for respondents aged 70 and older. EORTC QLQ-C30 summary scores also indicate that there is a statistically significant difference according to age, where scores are lower with increasing age.

Supplement Figure 1 shows mean scores of EORTC QLQ-C30 scores for all scales and items in general population according to age and gender. Men reported better general quality of life, as well as better physical and emotional functioning. There is a statistically significant difference if we compare the results for symptom scale and single items for fatigue, pain, dyspnoea and insomnia by gender, where the ranges are higher for women. Men reported better functioning for all items on the symptom scale. If we compare the results for single items, women reported less constipation, diarrhoea and financial problems. Supplement Figure 2 shows EORTC QLQ-C30 summary score in Croatian general population according to age and gender. Supplement Table 2 shows results for the EORTC QLQ-C30 for all scales and sin-
TABLE 2. Mean score (MS) and standard deviation (SD) for all EORTC QLQ-C30 dimensions and scales in general population and multiple myeloma (MM) patients at diagnosis at the time of setting the diagnosis

\begin{tabular}{lccccc}
\hline & \multicolumn{4}{c}{$\begin{array}{c}\text { MM patients } \\
\text { at diagnosis }\end{array}$} & \multicolumn{2}{c}{$\begin{array}{c}\text { General } \\
\text { population }\end{array}$} \\
\cline { 2 - 6 } & MS & SD & MS & SD & P* $^{*}$ \\
\hline Global health status/ quality of life & 58.9 & 19.4 & 68.2 & 20.6 & .003 \\
Physical functioning & 63.0 & 26.2 & 81.7 & 20.1 & .000 \\
Role functioning & 52.6 & 38.6 & 83.1 & 25.1 & .000 \\
Emotional functioning & 75.8 & 21.7 & 75.1 & 21.6 & .820 \\
Cognitive functioning & 82.0 & 22.0 & 83.5 & 20.1 & .725 \\
Social functioning & 60.1 & 34.0 & 87.4 & 20.6 & .000 \\
Fatigue & 41.0 & 24.3 & 31.9 & 23.4 & .014 \\
Nausea/vomiting & 7.5 & 13.9 & 6.2 & 13.3 & .346 \\
Pain & 38.2 & 33.4 & 26.1 & 26.5 & .020 \\
Dyspnoea & 27.5 & 29.6 & 19.7 & 25.2 & .078 \\
Insomnia & 31.4 & 32.9 & 28.3 & 30.9 & .556 \\
Appetite loss & 21.6 & 34.5 & 8.5 & 18.7 & .010 \\
Constipation & 13.7 & 25.9 & 10.1 & 20.8 & .392 \\
Diarrhoea & 9.8 & 20.3 & 7.7 & 16.2 & .712 \\
Financial difficulties & 39.2 & 36.9 & 10.6 & 22.6 & .000 \\
Summary score & 72.9 & 17.8 & 83.0 & 14.8 & .000 \\
\hline
\end{tabular}

*p = Mann-Whitney test

gle items according to a place of residence. There is a statistically significant difference in general health status according to a place of residence; the scores are higher for respondents from Istria and Medimurje, and lowest for respondents from Dalmatia. Furthermore, there is a statistically significant difference in physical functioning, role functioning, emotional functioning, cognitive functioning and social functioning according to a place of residence; the scores are again the highest for respondents from Istria and Medimurje.

Table 2 presents the comparison between general population and multiple myeloma patients for all EORTC QLQ-C30 dimensions. There is a statistically significant difference for global health status, physical functioning, physical functioning, role functioning, social functioning, fatigue, pain, appetite loss and financial difficulties. The values for global health status, physical functioning, role functioning and social functioning are higher in general population while values for fatigue, pain, appetite loss and financial difficulties are higher in MM patients.

There is a statistically significant difference in physical functioning, cognitive functioning, social 
TABLE 3. Mean score (MS) and standard deviation (SD) for all EORTC QLQ-C30 dimensions and scales in multiple myeloma (MM) patients before and after chemotherapy and transplantation

\begin{tabular}{|c|c|c|c|c|c|c|c|c|c|c|}
\hline & \multicolumn{2}{|c|}{$\begin{array}{c}\text { MM-before } \\
\text { chemotherapy }\end{array}$} & \multicolumn{2}{|c|}{$\begin{array}{c}\text { MM-after } \\
\text { chemotherapy }\end{array}$} & $\mathrm{p}^{*}$ & \multicolumn{2}{|c|}{$\begin{array}{c}\text { MM-before } \\
\text { transplantation }\end{array}$} & \multicolumn{2}{|c|}{$\begin{array}{c}\text { MM-after } \\
\text { transplantation }\end{array}$} & $\mathrm{p}^{*}$ \\
\hline Global health status/ quality of life & 53.3 & 21.4 & 49.7 & 22.5 & .283 & 64.4 & 15.7 & 74.7 & 18.0 & .008 \\
\hline Physical functioning & 53.9 & 26.3 & 38.9 & 21.3 & .011 & 71.8 & 23.4 & 71.8 & 19.1 & .955 \\
\hline Role functioning & 50.7 & 39.8 & 46.7 & 31.9 & .458 & 54.5 & 38.2 & 77.6 & 24.5 & .009 \\
\hline Emotional functioning & 73.3 & 27.6 & 78.0 & 21.9 & .776 & 78.2 & 14.5 & 84.3 & 23.3 & .107 \\
\hline Social functioning & 57.3 & 37.6 & 787 & 26.6 & .007 & 62.8 & 30.7 & 78.0 & 26.1 & .026 \\
\hline Fatigue & 43.1 & 27.3 & 64.0 & 22.3 & .001 & 39.1 & 21.5 & 40.2 & 24.6 & .782 \\
\hline Nausea/vomiting & 7.3 & 15.3 & 5.3 & 13.4 & .603 & 7.7 & 12.7 & 0.0 & 0.0 & .010 \\
\hline Pain & 39.3 & 36.9 & 54.7 & 25.2 & .039 & 37.2 & 30.3 & 31.4 & 28.0 & .323 \\
\hline Constipation & 16.0 & 32.1 & 20.0 & 28.9 & .564 & 11.5 & 18.7 & 1.3 & 6.5 & .011 \\
\hline Diarrhoea & 9.3 & 22.6 & 6.7 & 19.2 & .414 & 10.3 & 18.3 & 1.3 & 6.5 & .035 \\
\hline Financial diffuclities & 41.3 & 41.1 & 14.7 & 28.9 & .005 & 37.2 & 33.1 & 20.5 & 29.9 & .018 \\
\hline Summary score & 69.6 & 19.5 & 67.7 & 14.5 & .181 & 76.0 & 15.7 & 83.3 & 9.7 & .097 \\
\hline
\end{tabular}

${ }^{*} \mathrm{p}=$ Wilcoxon signed rank test

functioning, fatigue, pain, insomnia and financial difficulties in the measurements before and after chemotherapy where the results are worse in measurements after chemotherapy. Furthermore, there is statistically significant difference for global health status, role functioning, social functioning, nausea and vomiting, diarrhoea and financial difficulties in measurements after transplantation. For global health status in post-testing positive ranks in 15 cases were observed which means that they indicate better results. For role functioning positive ranks in 15 cases were observed while in social functioning positive ranks in 14 cases were observed which means that they indicated worse results. While for nausea and vomiting in 8 cases, constipation in 7 cases, diarrhoea in 7 cases and financial difficulties in 12 cases negative ranks were observed which means that they indicate better results (Table 3 ).

When analysing QLQ-MY20 questionnaire, there is a statistically significant difference for body image and disease symptoms in measurements before and after chemotherapy. In post-testing negative ranks in 7 cases for body image were observed which means that they indicate better results. For disease symptoms in post-testing positive ranks in 16 cases were observed which means that the values were higher in post-testing in accordance to initial

TABLE 4. Mean score (MS) and standard deviation (SD) for all QLQ-MY20 scales in multiple myeloma (MM) patients before and after chemotherapy and transplantation

\begin{tabular}{|c|c|c|c|c|c|c|c|c|c|c|}
\hline & \multicolumn{2}{|c|}{$\begin{array}{c}\text { MM-before } \\
\text { chemotherapy }\end{array}$} & \multicolumn{2}{|c|}{$\begin{array}{c}\text { MM-after } \\
\text { chemotherapy }\end{array}$} & $p^{*}$ & \multicolumn{2}{|c|}{$\begin{array}{c}\text { MM-before } \\
\text { transplantation }\end{array}$} & \multicolumn{2}{|c|}{$\begin{array}{c}\mathrm{MM} \text {-after } \\
\text { transplantation }\end{array}$} & $\mathrm{p}^{*}$ \\
\hline Future perspective & 2.2 & 0.7 & 2.0 & 0.7 & .285 & 2.2 & 0.7 & 1.8 & 0.7 & .036 \\
\hline Body image & 1.9 & 1.3 & 1.4 & 0.9 & .023 & 1.4 & 0.6 & 1.4 & 0.6 & 1.00 \\
\hline Disease symptoms & 1.9 & 0.6 & 2.2 & 0.6 & .039 & 1.7 & 0.6 & 1.8 & 0.7 & .571 \\
\hline Side effects of treatment & 2.1 & 0.7 & 1.8 & 0.4 & .361 & 1.9 & 0.6 & 1.5 & 0.3 & .144 \\
\hline
\end{tabular}

${ }^{*} \mathrm{p}=$ Wilcoxon signed rank test 
testing. Furthermore, there is a statistically significant difference for future perspective in measurements before and after transplantation (Table 4).

We investigated the ability of the QLQ-C30 summary score to distinguish between groups formed according to treatment choice (chemotherapy, transplantation) and general population status. Using the treatment choice as a variable, the QLQ-C30 summary score showed the changes from the initial testing to post-testing. For MM patients on chemotherapy, results showed that in comparison to general population and transplanted patients, their functional scale was worse in the initial testing and it further deteriorated in post-testing. For transplanted patients the results showed worse results in comparison to general population but better when compared to patients on chemotherapy. In post-testing the results were improved. Three months after treatment the summary score was comparable to general population and significantly better when compared to patients on chemotherapy.

\section{Correlation between EORTC QLQ-C30 and QLQ-MY20 in MM patients before treatment}

The correlations between the EORTC QLQ-C30 dimensions and scales and 4 items of the QLQ-MY20 before treatment are shown in Table 5 for patients on chemotherapy, and Table 6 for transplanted patients. The strongest positive correlation was found for pain (0.779) and disease symptoms, and strongest negative correlation for fatigue $(-0.808)$ and physical functioning for patients on chemotherapy. Furthermore, for transplanted patients the strongest positive correlation was found for pain (0.707) and disease symptoms, and strongest negative correlation for physical functioning $(-0.710)$ and side effects of treatment.

\section{Discussion}

Quality of life is now widely recognized as a central outcome of many clinical trials. ${ }^{3}$ It is a multi-dimensional concept based on a holistic view of human well-being. It considers a number of domains of people's lives and the interplay between these dimensions. The principal domains include: subjective well-being, health, income and standard of living, relationship with family and friends, work and quality of jobs, sense of inclusion in one's local community, and personal safety. ${ }^{24}$
In this study we presented the reference data of the EORTC QLQ-C30 quality of life dimensions for the general Croatian population. This study begun with the aim of obtaining reference data of the EORTC QLQ-C30 quality of life dimension in a sample of Croatian adults older than 40 years who were not suffering from a malignant disease. In Croatia, there is around $30 \%$ of population younger than 40 years, however there are only around $4 \%$ of cancer patients that are diagnosed below this age. ${ }^{1}$ The collected data are transformed into expected mean HRQL scores for distinctive demographic population groups.

Our results illustrate that mean scores vary with age, gender and place of residence. Men reported better general health status as well as physical and emotional functioning. On the symptom scale they reported less symptoms when compared to women. Women reported significantly higher scores for fatigue, pain, dyspnoea and insomnia. These findings are similar to those of the Norwegian study, which was the first to be conducted in a general population. Their study also showed that men reported fewer symptoms and better quality of life on all scales. ${ }^{25}$ Moreover, a study conducted in German general population showed that men reported better quality of life on all scales and fewer symptoms in comparison to women. ${ }^{5}$ The first study conducted on a South-eastern European population was in neighbouring Slovenia, also showed that men report better quality of life on the majority of specific scales and that they report fewer symptoms, but the results were mostly not statistically significant. ${ }^{15}$

Our study also highlights that all scores deteriorated with age and older respondents reported more symptoms present. Scores were statistically higher for respondents 70 and more years old for physical, emotional and social functioning. The study conducted in the Norwegian population also showed that increasing age influences the scores; both QoL scales and all functional scales, except for emotional, showed a gradual decline in mean scores with increasing age for both sexes. ${ }^{25}$ The study conducted in Slovenia also showed that older participants report more symptoms. ${ }^{15}$ For general health status the ranges are interestingly highest for the age group 50-59 in the Croatian population.

The results indicate that place of residence has great impact on quality of life. Two of the areas in Croatia report better global functioning and less symptoms present. The ranges for physical, emotional, cognitive and social functioning are the highest for the respondents from Istria and 
TABLE 5. Correlation between dimensions and scales in the EORTC QLQ-C30 and QLQ-MY20 (chemotherapy)

\begin{tabular}{|c|c|c|c|c|c|c|c|c|c|c|c|c|c|c|c|c|c|c|c|}
\hline & & & & ET & HS & & & & CF & SF & $F$ & NV & $\mathbf{P}$ & D & 1 & AL & C & A & D \\
\hline FP & 1 & & & & & & & & & & & & & & & & & & \\
\hline BI & & 1 & 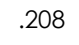 & & & & 333* & & & -.396 & ום & $*$ & .164 & -.044 & 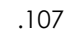 & 36 & .074 & 9 & 70 \\
\hline DS & $30 *$ & .208 & 1 & .669 & -.459 & 8 & -.429 & 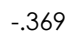 & 5 & 0 & 42 & $9^{*}$ & .779 & 5 & .127 & .512 & 50 & .274 & 243 \\
\hline SET & $395^{*}$ & 86 & 669 & 1 & -.277 & -.513 & -.462 & -.579 & -.577 & -.568 & .560 & 599 & 48 & .116 & .30 & .644 & . .121 & $45]$ & 169 \\
\hline GHS & 213 & & -.459 & -.277 & 1 & ${ }^{\prime}$ & $.341^{*}$ &. & $.336^{*}$ & .468 & -.399 & -.205 & 2* & . & .0 & -.528 & -.033 & -.489 & -.335 \\
\hline PF & 84 & 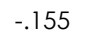 & -.678 & -.513 & .427 & 1 & .588 & .3 & 524 & .430 & -.8 & -.211 & 5 & & .165 & -.389 & 56 & 36 & -.147 \\
\hline RF & -.443 & 33* & -.429 & -.462 & .34 & .588 & 1 & .384 & $.359 *$ & .49 & -.564 & -.373 & -.554 & -.376 & -.094 & .449 & .113 & .259 & $-.316^{\circ}$ \\
\hline EF & -.478 & -428 & -.369 & -.579 & .503 & .367 & .384 & 1 & $.329^{*}$ & .692 & -.266 & 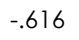 & & & .048. & -.596 & -.117 & -.424 & $-4] 4$ \\
\hline CF & 057 & & -.545 & -.577 & $.336^{*}$ & .027 & $.359^{*}$ & $.329^{*}$ & 1 & .176 & -.625 & ${ }^{\circ}$ & & & 8 & & .043 & & \\
\hline SF & 410 & 90 & -.400 & - & .4 & .4 & .491 & .6 & .110 & 1 & -.2 & -.498 & -.3 &.- & .00 & -.589 & .06 & & -.5 \\
\hline$F$ & 086 & 131 & .642 & .56 & -399 & -807 & -564 & -266 & 625 & 22 & & .272 & 71 & 45 & .066 & 37 & .127 & 3 & 119 \\
\hline NV & & & 288 & .5 & -.205 & -.277 & & & & & & 1 & & & .0 & & & & \\
\hline $\mathbf{P}$ & $.322^{*}$ & .164 & .779 & .48 & $332 *$ & -725 & & -.4 & -.502 & & & .2 & & & -.1 & & & & \\
\hline D & .119 & -.044 & 2 & 1 & $-.283^{*}$ & . & - . & .115 & -.170 & -.134 & .456 & -1 & $.350 *$ & & .2 & .078 & -.187 & & .160 \\
\hline 1 & -.104 & .107 & -.12 & .30 & .010 & .165 & -.0 & .0 & -.0 & .00 & .0 & .08 & -.1 & .2 & 1 & .23 & -.186 & .271 & .194 \\
\hline$A L$ & $.309 *$ & .186 & .512 & .044 & -.528 & .389 & -.449 & -.596 & -.424 & -.589 & .374 & .605 & .389 & .078 & .238 & & .000 & $.329 *$ & .375 \\
\hline C & .004 & -.074 & .150 & -.121 & -.033 & -.156 & -.113 & 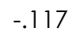 & 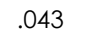 & 64 & .127 & .12 & .161 & -.187 & -.186 & 0 & & -.089 & -.1 \\
\hline DIA & 17 & .2 & 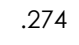 & .45 & -2 & -.136 & -.259 & 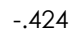 & $-.349^{*}$ & -.3 & .369 & .36 & .2 & .2 & .2 & $.329 *$ & , & 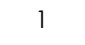 & .517 \\
\hline $\mathrm{FL}$ & 264 & .270 & 243 & .469 & $335^{*}$ & .147 & $316^{*}$ & -.414 & -.262 & -.534 & .119 & $.317^{*}$ & .18 & .160 & .19 & (N) & -.133 & 517 & 1 \\
\hline
\end{tabular}

$\mathrm{AL}=$ appetite loss; $\mathrm{BI}=$ body image $; \mathrm{C}=$ constipation; $\mathrm{CF}=$ cognitive functioning; $\mathrm{D}=$ dyspnoea; $\mathrm{DIA}=$ diarrhoea; $\mathrm{DS}=$ disease symptoms; $\mathrm{EF}=$ emotional functioning; $\mathrm{F}=$ fatigue $\mathrm{FD}=$ financial difficulties; $\mathrm{FP}=$ future perspective; $\mathrm{GHS}=$ global health status; I = insomnia; $\mathrm{NV}=$ nausea/vomiting; $\mathrm{P}=\mathrm{pain} ; \mathrm{PF}=\mathrm{physical}$ functioning; $\mathrm{RF}=$ role functioning; $\mathrm{SET}=$ side effects of treatment; $\mathrm{SF}=$ social functioning; * Correlation is significant at the 0.05 level (2-tailed)

TABLE 6. Correlation between dimensions and scales in the EORTC QLQ-C30 and QLQ-MY20 (transplantation)

\begin{tabular}{|c|c|c|c|c|c|c|c|c|c|c|c|c|c|c|c|c|c|c|c|}
\hline & FP & BI & DS & SET & GHS & PF & RF & EF & $\mathrm{CF}$ & SF & $F$ & NV & $\mathbf{P}$ & D & I & $A L$ & C & DIA & FD \\
\hline FP & 1 & $.451^{* *}$ & .029 & .289 & -.383 & -.246 & -.203 & -.499 &,- 106 & $-.333^{*}$ & $.287^{*}$ & .238 & -.035 & .145 & $.296^{*}$ & .371 & $.309^{*}$ & .049 & .053 \\
\hline BI & .451 & 1 & -.142 & .026 & -.109 & -.047 & -.009 & -.083 & , 105 & .016 & .038 & -.006 & -.151 & -.048 & $.333^{*}$ & .109 & .053 & -.029 & -.195 \\
\hline DS & .029 & -.142 & 1 & .4 & $z^{*}$ & 589 & 19 & * & ,228 &.- & .527 & -.051 & 7 & .406 & 145 & .035 & .060 & 72 & 188 \\
\hline SET & .289 & .026 & .447 & 1 & -.615 & -.710 & -.627 & -.3 &,- 299 & -.455 & .570 & .597 & .519 & .642 & $.377^{*}$ & .523 & .470 & 485 & $.337^{*}$ \\
\hline GHS & -.383 & -.109 & $-.312^{*}$ & -.615 & 1 & .629 & .518 & $.349^{*}$ & , 159 & .498 & -.436 & -.467 & -.423 & -.443 & -.071 & -.192 & -.429 & -.371 & -.370 \\
\hline PF & -.246 & -.047 & -.589 & -.710 & .629 & 1 & .562 & .403 & , 147 & .506 & -.645 & -.375 & -.643 & -.447 & -.379 & $-.325^{*}$ & -.232 & -.171 & -.443 \\
\hline RF & -.203 & -.009 & -.449 & -.627 & .518 & .562 & 1 & .346 &,- 015 & .580 & -.563 & $-.341^{*}$ & -.626 & -.403 & -.194 & -.200 & -.209 & -.127 & -.421 \\
\hline EF & -.499 & -.083 & $-.339^{*}$ & -.397 & $.349^{*}$ & .403 & $.346^{*}$ & 1 & , 188 & .378 & $-.347^{*}$ & -.217 & -.146 & -.191 & -.395 & -.120 & -.123 & -.054 & -.258 \\
\hline $\mathrm{CF}$ & -.106 & .105 & .228 & -.299 & .159 & .147 & -.015 & .188 & 1 & .024 & -.002 & $-.348^{*}$ & .023 & -.041 & -.105 & -.033 & $-.304^{*}$ & -.143 & -.250 \\
\hline SF & $-.333^{*}$ & .016 & -.397 & -.455 & .498 & .506 & .580 & .378 & ,024 & 1 & -.539 & -.245 & -.376 & -.178 & -.188 & -.078 & -.158 & -.080 & $-.292^{*}$ \\
\hline$F$ & $.287^{*}$ & .038 & .527 & .570 & 36 & 645 & 563 & $-.347^{*}$ &,- 002 &.- & 1 & .29 & .563 & $.294^{*}$ & .423 & .44 & .154 & .266 & $.336^{*}$ \\
\hline NV & 238 & -.006 & -.051 & .597 & -.467 & -.375 & $-.341^{*}$ & -.217 &,$- 348^{*}$ & -.245 & $.299^{*}$ & 1 & .258 & .263 & .197 & .587 & .660 & .464 & $.299^{*}$ \\
\hline $\mathbf{P}$ & -.035 & -.151 & .707 & .519 & -.423 & -.643 & -.626 & -.146 & ,023 & -.376 & .563 & .258 & 1 & .563 & .077 & .182 & .188 & .143 & .376 \\
\hline D & .145 & -.048 & .406 & .642 & -.443 & -.447 & -.403 & -.191 &,- 041 & -.178 & $.294^{*}$ & .263 & .563 & 1 & .149 & $.346^{*}$ & .190 & .356 & .178 \\
\hline I & $.296^{*}$ & $.333^{*}$ & .145 & $.377^{*}$ & -.071 & -.379 & -.194 & -.395 &,- 10 & -.188 & .42 & .1 & .0 & .14 & 1 & .398 & .123 & .264 & $.288^{*}$ \\
\hline$A L$ & $.371^{* *}$ & .109 & .035 & .523 & -.192 & $-.325^{*}$ & -.200 & -.120 &,- 033 & -.078 & .441 & .587 & .182 & $.346^{*}$ & .398 & 1 & .271 & .363 & .148 \\
\hline C & $.309^{*}$ & .053 & -.060 & .470 & -.429 & -.232 & -.209 & -.123 &,$- 304^{*}$ & -.158 & .154 & .660 & .188 & .190 & .123 & .271 & 1 & .540 & .152 \\
\hline DIA & .049 & -.029 & -.072 & .485 & -.371 & -.171 & -.127 & -.054 &,- 143 & -.080 & .266 & .464 & .143 & .356 & .264 & .363 & .540 & 1 & .104 \\
\hline FD & .053 & -.195 & .188 & $.337^{*}$ & -.370 & -.443 & -.421 & -.258 &,- 250 & $-.292^{*}$ & $.336^{*}$ & $.299^{*}$ & .376 & .178 & $.288^{*}$ & .148 & .152 & .104 & 1 \\
\hline
\end{tabular}

$\mathrm{AL}=$ appetite loss $; \mathrm{BI}=$ body image $; \mathrm{C}=$ constipation; $\mathrm{CF}=$ cognitive functioning; $\mathrm{D}=$ dyspnoea; $\mathrm{DIA}=$ diarrhoea; $\mathrm{DS}=$ disease symptoms; $\mathrm{EF}=$ emotional functioning; $\mathrm{F}=$ fatigue $\mathrm{FD}$ = financial difficulties; $\mathrm{FP}=$ future perspective; $\mathrm{GHS}=$ global health status; $\mathrm{I}=$ insomnia; $\mathrm{NV}=$ nausea/vomiting; $\mathrm{P}=\mathrm{pain} ; \mathrm{PF}=\mathrm{physical}$ functioning; $\mathrm{RF}=$ role functioning; $\mathrm{SET}=$ side effects of treatment; $\mathrm{SF}=$ social functioning: * Correlation is significant at the 0.05 level (2-tailed) 
Medimurje. It is hard to define what is the predictor for this result because one area is continental and the other is a coastal area. Both areas are developed and it could be that socio-economic circumstances in these areas have influenced the results. Development index is a composite indicator calculated as a customized average of standardized values of socio-economic indicators for measuring the degree of development in a given period. In Croatia, the following indicators are used to calculate the development index: average income per capita, average source income per capita, the average unemployment rate, movements of general population, degree of education of the population (tertiary education) and the aging index. Local self-government units are classified into categories considering development index. Third and fourth level represent above-average ranking units. Istria currently belongs to the forth level and Medimurje to the third level, which means that they are aboveaverage developed. ${ }^{26,27}$ For global health status the ranges are the lowest for respondents from Dalmatia.

In addition, in this study we also aimed to assess the impact of the disease and its primary systemic treatment on their HrQoL in a prospective cohort of multiple myeloma patients older than 40 years at the time of diagnosis. If we compare the results for general population and MM patients which were obtained using the EORTC QLQ-C30 questionnaire, it can be seen that during the initial testing patients suffering from multiple myeloma have lower scores for global health status, physical functioning, role functioning and social functioning, while they have higher scores for fatigue, pain, appetite loss and financial difficulties. It can be concluded that they have more symptoms present.

According to Delforge et al., about $70 \%$ of the patients suffer from pain at the time of diagnosis. ${ }^{28}$ Our results indicate that pain is a great predictor of global health status in general population, as well as in MM patients. In general population, pain and fatigue were the most expressed symptoms, while in MM patients pain, fatigue, insomnia, dyspnoea and appetite loss were the most expressed symptoms. Severity, type of disease, symptoms and treatment are important determinants of HrQoL in patients with multiple myeloma. Advanced disease and treatment related symptoms are associated with lower HrQoL. ${ }^{16}$

Our descriptive and exploratory analysis suggests a beneficial effect of transplanted patients three months after therapy on HrQoL. HrQoL scores were higher among transplanted patients, compared to those who underwent chemotherapy. Respondents who underwent chemotherapy were mostly older than 70 years with a different comorbidity. They also indicate that approximately 9 months passed from the occurrence of the first symptoms and diagnosis, due to the attribution of symptoms to other diseases, most commonly to spinal diseases. Also, patients starting treatment for the first time are affected by the psychological burden of their recent diagnosis and experience treatment related toxicities which they have not previously been exposed to. ${ }^{29}$ Furthermore, the period of three months was sufficient for transplanted patients to recover from high doses of chemotherapy that preceded transplantation. On the other hand, patients who underwent chemotherapy, regardless of the small dosage of therapy, noticed that they have deterioration in symptoms.

The Netherlands study, also conducted on 51 transplanted patients during the treatment and 12 months after, shown that after 12 months the results were better for physical, role, emotional, cognitive and social functioning. Additionally, for symptoms, the results were better for fatigue, pain and appetite loss while nausea/vomiting and diarrhoea deteriorated in results. ${ }^{30}$ Our results illustrate that scores for global health status, physical functioning, role functioning and cognitive functioning are higher three months after transplantation, while results for fatigue, pain, dyspnoea and constipation are higher after chemotherapy. Patients who underwent chemotherapy had more symptoms present three months after therapy in comparison to patients who underwent transplantation.

An additional valuable output from our study is the correlation analysis of the EORTC QLQ-C30 scores and 4 items of the EORTC QLQ-MY20. This method can provide reliable, accurate descriptions of the HrQoL of patients with multiple myeloma. ${ }^{29}$ Correlation analysis for patients on chemotherapy from our study shows that side effects of treatment strongly correlate with body image and disease symptoms; furthermore, physical functioning strongly correlates with disease symptoms, side effects of treatment and role functioning, while emotional functioning correlates with side effects of treatment and social functioning. Cognitive functioning strongly correlates with disease symptoms, side effects of treatment and physical functioning. When symptoms are taken into consideration fatigue correlates with disease symptoms, side effects of treatment, physical functioning and cognitive functioning, while pain strongly correlates with disease symptoms, physical functioning, 
role functioning, cognitive functioning and fatigue. Appetite loss strongly correlates with disease symptoms, side effects of treatment, global health status, emotional functioning, social functioning, and nausea/vomiting.

Correlation analysis for transplanted patients from our study shows that global health status strongly correlates with side effects of treatment; furthermore, physical functioning strongly correlates with disease symptoms, side effects of treatment and global health status but also with role functioning; while role functioning and physical functioning correlate with social functioning. When symptoms are taken into consideration the pain strongly correlates with the largest number of items. Pain strongly correlates with disease symptoms, side effects of treatment, physical functioning, role functioning and fatigue. Other symptoms correlate only with one item as follows: dyspnoea with side effects of treatment, appetite loss with nausea/vomiting, constipation with nausea/vomiting and diarrhoea with constipation.

The study conducted in France shows the strongest correlation between EORTC QLQ-C30 global health status and QLQ-MY20 disease symptoms. They also highlight that information such as this may be useful in studies of HrQoL in multiple myeloma by helping to establish the factors that have greatest influence on the global health status score. ${ }^{29}$ These data do not represent the course of an individual patient's disease. Nonetheless, they offer a basis for hypotheses regarding the factors that could influence HrQoL throughout the disease course. $^{29}$

Improvements in overall survival achieved in recent years with new therapies for MM patients are a great achievement. Data from clinical trials suggest that the benefits of multiple myeloma treatment may outweigh the negative effects of toxicities and disease progression. ${ }^{29}$ Quality of life is influenced by the ability to adapt to unfortunate conditions or it can be said that that changes in HQL scores are subordinate to the individual subjective assessment of life situations which they consider to be important. ${ }^{31,32}$ However, the updating and improving QoL measure is essential to remain relevant in new treatments. ${ }^{33,34}$ Quality of life could be monitored objectively, excluding the impact of important socio-demographic factors. ${ }^{15}$

In conclusion, this study is the first to present Croatian general population reference values for the EORTC QLQ-C30 questionnaire. Age, gender and place of residence are important predictors of quality of life in Croatian population. For MM patients, our study identified treatment effects consistent with those from other observational studies and provide new data on HrQoL across two different treatment choices for patients with multiple myeloma treated in Croatian clinical centres. According to available information there has been no research on MM patients before and after transplantation using the combination of EORTC QLQ-C30 and EORTC MY-20 questionnaires. It was recognized once again, that there is a need to compare the patient's quality of life to the quality of life in the general population.

Similarly as in the same research in our neighbouring Slovenia, ${ }^{15}$ our results are applicable to more than $95 \%$ of the entire pool of Croatian cancer patients. Still, the HrQoL in Croatian general population could be further explored on larger sample sizes, and also with a wider range of age groups, where younger respondents could also be included. This might improve the applicability in patients that are diagnosed as children, adolescents or young adults, especially given the sociodemographic and political changes that have taken place in Croatia over the past 30 years.

\section{Acknowledgements}

We would like to express our gratitude to all of participants who were engaged in research. Special thanks to the health team from the Clinical Hospital Dubrava and Clinical Hospital Merkur for their understanding and facilities that allowed completion of all data.

\section{References}

1. Croatian Institute of Public Health, Croatian National Cancer Registry. Cancer incidence in Croatia 2016. Bulletin No. 41. Zagreb: Croatian Institute of Public Health; 2019.

2. King MT. The interpretation of scores from the EORTC quality of life questionnaire QLQ-C30. Qual Life Res 1996; 5: 555-67. doi: 10.1007/BF00439229

3. Ford ME, Havstad SL, Kart CS. Assessing the reliability of the EORTC QLQ C-30 in a sample of older African and Caucasian adults. Qual Life Res 2001; 10: 533-41. doi: 10.1023/A:1013003014340

4. WHOQOL Group. Study protocol for the World Health Organization project to develop a quality of life assessment instrument. Qual Life Res 1993; 2: 153-9. doi: 10.1007/BF00435734

5. Schwarz R, Hinz A. Reference Dana for the quality of life questionnaire EORTC QLQ-C30 in the general German population. Eur J Cancer 2001; 37: 1345-51. doi: 10.1016/s0959-8049(00)00447-0

6. Sherman AC, Simonton S, Latif U, Plante TG, Anaissie EJ. Changes in quality of life and psychosocial adjustment among MM patients treated with high dose malphalan and autologous stem cell transplantation. Biol Blood Marrow Transplant 2009; 15: 12-20. doi: 10.1016/j.bbmt.2008.09.023 
7. Juul T, Petersen MA, Holzner B, Laurberg S, Christensen P, Gronvold M Danish population- based reference data for the EORTC QLQ-C30: associations with gender, age and morbidity. Qual Life Res 2014; 23: 2183-93. doi 10.1007/s11136-014-0675-y

8. Luckett T, King MT, Butow PN, Oguchi M, Rankin N, Price MA, et al. Choosing between the EORTC QLQ-C3O and FACT-G for measuring health- related quality of life in cancer clinical research: issues, evidence and recommendations. Ann Oncol 2011; 22: 2179-90. doi: 10.1093/annonc/mdq721

9. Cocks K, King MT, Velikova G, Martyn St-James M, Fayers PM, Brown JM. Evidence-based guidelines for determination of sample size and interpretation of the European Organization for the Research and Treatment of Cancer Quality of Life Questionnaire Core 30. J Clin Oncol 2011; 29: 89-96. doi: 10.1200/JCO.2010.28.0107

10. Aaronson NK, Ahmedzai S, Bergman B, Bullinger M, Cull A, Duez NJ, et al. The European Organization for Research and Treatment of Cancer QLQ-C30: A quality of life instrument for use in international clinical trials in oncology. $J$ Natl Cancer Inst 1993; 85: 365-76. doi: 10.1093/jnci/85.5.365

11. European Organization for Research and Treatment of Cancer. Questionnaires. [cited 2016 Jan 10]. Available at: https://qol.eortc.org/ questionnaire/qlq-my20/

12. European Organization for Research and Treatment of Cance Questionnaires (10.01.2016.) Available at: https://qol.eortc.org/questionnaire/eortc-qlq-c30/

13. Lonneke V, van de Poll-Franse LV, Mols F, Gundy CM, Creutzberg CL, Nout RA, et al. Normative data for the EORTC QLQ-C3O and EORTC- sexuality items in the general Dutch population. Eur J Cancer 2011; 47: 667-75. doi: 10.1016/j.ejca.2010.11.004

14. Michelson $\mathrm{H}$, Bolund $\mathrm{C}$, Nilsson B, Brandberg $\mathrm{Y}$. Health-related quality of life measured by the EORTC QLQ-C30. Reference values from a large sample of the Swedish population. Acta Oncol 2009; 39: 477-84. doi: $10.1080 / 028418600750013384$

15. Velenik V, Secerov-Ermenc A, But-Hadzic J, Zadnik V Health-related quality of life assessed by the EORTC QLQ-C30 questionnaire in the general Slovenian population. Radiol Oncol 2017; 51: 342-50. doi: 10.1515/ raon-2017-0021

16. Slovacek L, Slovackova B, Blazek M, Jebavy L. Quality of life in patients with multiple myeloma and malignant lymphoma undergoing autologous progenitor stem cell transplantation: the effect of selected psychosocial and health aspects on quality of life: a retrospective analysis. Rep Pract Oncol Radiother 2007; 12: 101-8. doi: 10.1016/S1507-1367(10)60046-6

17. SurveyMonkey. Sample Size Calculator. Calculate your sample soize. [cited 2019 Jan 15]. Available at: https://www.surveymonkey.com/mp/samplesize-calculator

18. Fayers P, Aaronson NK, Bjordal K, Sullivan M. EORTC QLQ-C3O scoring manual. Brussels: EORTC Publications; 1997.

19. Tkalac Verčič A, Sinčić Ćorić D, Pološki Vokić N. Research methodology manual -How to design, implement and describe scientific and professional research. Zagreb: M.E.P. d.o.o; 2012.

20. Petz B, Kolesarić V, Ivanec D. [Petzova statistics]. [Croatian]. Zagreb: Naklada Slap; 2012.

21. Šošić I. [Applied statistics 2]. [Croatian]. Updated edition. Zagreb: Školska knjiga; Zagreb 2006.

22. Nielsen LK, Abildgaard N, Jarden M, Wirenfeld Klausen T. Methodological aspects of health-related quality of life and analysis in patients with multiple myeloma. Br J Haematol 2019; 185: 1-14. doi: 10.1111/bjh.15759

23. Giesinger JM, Kieffer JM, Fayers PM, Groenvold M, Petersen MA, Scott NW, et al. Replication and validation of higher order models demonstrated that a summary score for the EORTC QLQ-C30 is robust. J Clin Epidemiol 2016; 69: 79-88. doi: 10.1016/j.jclinepi.2015.08.007

24. Bejaković $P$, Kaliterna Lipovčan $\amalg$. Quality of life in Croatia: key findings from national research. Dublin: European Foundation for the Improvement of Living and Working Conditions; 2007.

25. Hjermstad MJ, Fayers PM, Bjordal K, Kaasa S. Health related quality of life in the general Norwegian population assessed by the European Organization for Research and Treatment of Cancer Core Quality -of-Life Questionnaire: The QLQ=C30 (+3). J Clin Oncol 1998; 16: 1188-96. doi: 10.1200/JCO.1998.16.3.1188
26. Ministry of Regional Development and European Union Funds. Development index. [cited 2018 Jun 21]. Available at: https://razvoj.gov.hr/o-ministarstvu/ regionalni-razvoj/indeks-razvijenosti/112

27. Government of Republic of Croatia. Act on Regional Development of the Republic of Croatia 147/14, 123/17. NN. 132/2017. [cited 2018 Jun 21] Available at: https://www.zakon.hr/z/239/Zakon-o-regionalnom-razvojuRepublike-Hrvatske.

28. Delforge M, Minuk L, Eisenmann JC, Arnulf B, Canepa L, Fragasso A, et al. Health-related quality of life in patients with newly diagnosed multiple myeloma in the FIRST trial: lenalidomide plus low-dose dexamethasone versus melphalan, prednisone, thalidomide. Haematologica 2015; 100: 826-33. doi: 10.3324/haematol.2014.120121

29. Despiegel N, Touboul C, Flinois A, Saba G, Suzan F, Gonzales-McQuire S Health-related quality of life of patients with multiple myeloma treated in routine clinical practice in France. Clin Lymphoma Myeloma Leuk 2018; 19: 13-28. doi: 10.1016/j.clml.2018.08.01

30. Uyl-de Groot CA, Buijt I, Gloudemans IJM, Ossenkoppele GJ, Van den Berg $\mathrm{HP}$, Huijgens PC. Health related quality of life in patients with multiple myeloma undergoing a double transplantation. Eur J Haematol 2005; 74: 136-43. doi: 10.1111/j.1600-0609.2004.00346.x

31. Osoba D, Rodrigues G, Myles J, Zee B, Pater J. Interpreting the significance of changes in health-related quality of life scores. J Clin Oncol 1998; 16: 139-44. doi: 10.1200/JCO.1998.16.1.139

32. Gjural S, Conroy T, Fleissner C, Sezer O, King PM, Avery KN, et al. Assessing quality of life in patients with colorectal cancer: An update of the EORTC quality of life questionnaire. Eur J Cancer 2007; 43: 1564-73. doi: 10.1016/j. ejca.2007.04.005

33. Lange MM, Marijnen CAM, Maas CP, Putter $\mathrm{H}$, Rutten HJ, Stiggelbout AM, et al. Risk factors for sexual dysfunction after rectal cancer treatment. Eur J Cancer 2009; 45: 1578-88. doi: 10.1016/j.ejca.2008.12.014

34. Salaffi F, Carotti M, Gasparini S, Intorcia M, Grassi W. Health and Quality of Life Outcomes. The health-related quality of life in rheumatoid arthritis, ankylosing spondylitis, and psoriatic arthritis: a comparison with a selected sample of healthy people. Health Qual Life Outcomes 2009; 18: 7-25. doi:10.1186/1477-7525-7-25 